\title{
MEMRISTOR - THE FOURTH FUNDAMENTAL ELEMENT
}

\author{
Valeri Mladenov \\ Technical University of Sofia, \\ Department Theoretical Electrical Engineering, \\ 8, St. Kliment Ohridski Blvd, 1756 Sofia, Bulgaria, \\ e-mail:valerim@tu-sofia.bg
}

\begin{abstract}
This paper presents a brief overview of the fourth fundamental one-port element - the memristor. The theoretical fundaments of the idealized memristor predicted by Chua are represented. The main memristor realizations based on different materials are considered. Special attention is paid to their properties, memory effect, principles of operation and important applications in electronic devices, as reconfigurable circuits, memories, neural networks, logic gates and others. Due to the high complexity of the processes in the memristors their modeling is substantial and plays important role in memristor circuit design. A comparison of their main models is done, paying attention to their advantages, exploration for their new potential applications and the need of representation the nonlinear ionic drift for high-level and highfrequency signals.

Keywords: memristors, nonlinear ionic drift, modeling, window function.
\end{abstract}

\section{INTRODUCTION}

The resistance-switching phenomenon, observed in many amorphous metal oxides and transient metal chemical oxides, such as $\mathrm{SiO}_{2}, \mathrm{Al}_{2} \mathrm{O}_{3}, \mathrm{Ta}_{2} \mathrm{O}_{5}, \mathrm{TiO}_{2}$, $\mathrm{HfO}_{2}$ and others has been analyzed since 1970 [1]. It has been established that such materials placed in a sandwiched metal-oxide-metal microstructure have the ability of altering their resistance in accordance with the applied voltage and memorizing their state for a very long-time interval after turning the sources off $[1,2]$. Similar unusual behavior has been predicted for the idealized memristor element by Leon Chua in 1971 [3]. The idealized memristor element is proposed in accordance with symmetry considerations and the dependencies between the four basic electric quantities (current $i$, voltage $v$, charge $q$ and

DOI: 10.7546/EngSci.LVII.20.01.01

Engineering Sciences, LVII, 2020, No. 1 
magnetic flux $\Psi)$ [3]. The proposed fourth basic one-port element relates the flux linkage $\Psi$, expressed as a time integral of the voltage, to the electric charge $q$, equal to the time integral of the current. The relations between the main electrical quantities and the fundamental one-port elements $[3,4]$ are described by two definition expressions (for the charge-current and fluxvoltage relations) and by the passive two-terminal basic elements - resistor, inductor, capacitor and memristor.

The memristor has the important capability of memorizing the electric charge moving through its cross-section and its resistance $M$, called also memristance, when the electrical signals are switched off $[2,4]$. Its current-voltage relationship that will be discussed in detail in the next Section is a pinched hysteresis loop, which shape and range depend on both the magnitude and the frequency of the applied signal $[3,4,5]$. Since the memristor element could remember its resistance after turning the sources off, then the memristor could be used as a non-volatile memory element $[2,3,6]$. The memory effect is founded on accumulating electric charges (positively charged particles - ions) in the memristor structure and retaining them when the memristor voltage is zero [2]. Several basic types of real physical memristors exist $[7,8,9,10,11]$. They are based on different chemical and physical structures and have different principles of operation. Titanium dioxide memristor is one of the most important nano-structured elements which still have extended applications $[3,4,12]$ and due to this it is a central object investigated in detail in the present paper. The polymeric memristors [7] are based on organic plastic materials. Single or parcels of molecules in such materials are able to conduct and switch currents and memorize information using electric charge accumulation [7]. Resistance switching has been also observed in many sandwich-like structures consisting of ferroelectric thin layers $[10,13]$. Their resistance can be reversibly changed between its limiting values for OFF state and ON state by applying an external signal. The polarization transfer has good stability for chemical alteration and is principally rapid, the interfacial resistance-switching phenomenon is optimistic to the new ferroelectric memristors [10, 13]. The spintronic memristor device is based on a thin oxide layer placed between two regions with good magnetic properties [10,14]. Principally, all types of memristor elements could be described using a system of two equations: an equation describing the current-voltage relationship, dependent on the memristor state, and another equation relating the time derivative of the state variable and the current $[3$, $8,10]$.

The modeling of the real memristors is a very important procedure due to the specific description of the different electric properties of the respective 
materials and their behavior in electric circuits $[15,16,17,18]$. According to the specific structure of the physical memristors several different models are proposed in the scientific literature $[15,16,17,19]$. Several of them have disadvantages mainly associated with the impossibility for realistic representation the nonlinear dopant drift for high level signals, unsuitability for operation and resistance switching at high-frequency and high-level voltages, high complexity, convergence problems and others $[16,19]$. These facts are responsible for the need of memristor models modifications and improvements [10]. In several modified by the author models $[10,20]$ in the respective window functions a voltage-dependent exponent and additional sine component are applied for more realistic representation of the nonlinear ionic dopant drift and of the switching properties for high-level and high-frequency electric signals. For increasing the stability and avoiding convergence problems, especially in SPICE environment, in a standard memristor model [9] and in a modified by the author memristor model [21] the non-differentiable step function is replaced by its differentiable and continuous analogues - functions based on exponential and tangent hyperbolic functions, respectively. In the scientific literature a complete comparison of the existing memristor models is not presented.

The motivation for this article is to present a detailed comparison of the main standard and the modified by the author memristor models according to different criteria, as switching speed, representation the boundary effects and the nonlinearity of the ionic dopant drift, and others, paying attention to their advantages and specific applications - in reconfigurable analog and digital devices, computer nonvolatile memories, neural networks, in-memory computing schemes and other electronic circuits, and searching for new possible future applications of the memristor elements.

The rest of the paper is organized as follows. Section 2 represents a theoretical fundament for the idealized, standard and physical memristors, paying attention on the differences between them and their specific present and new potential applications in electronic devices. In Section 3 a review and comparison of the basic standard and modified by the author memristor models are presented. The concluding notes are given in Section 4 .

\section{GENERAL INFORMATION FOR THE IDEALIZED AND THE PHYSICAL MEMRISTORS AND APPLICATIONS}

\subsection{The idealized memristor element}

The relationships between the basic electric quantities (current, voltage, charge and magnetic flux) are presented in Fig. 1(a). The memristor relates 


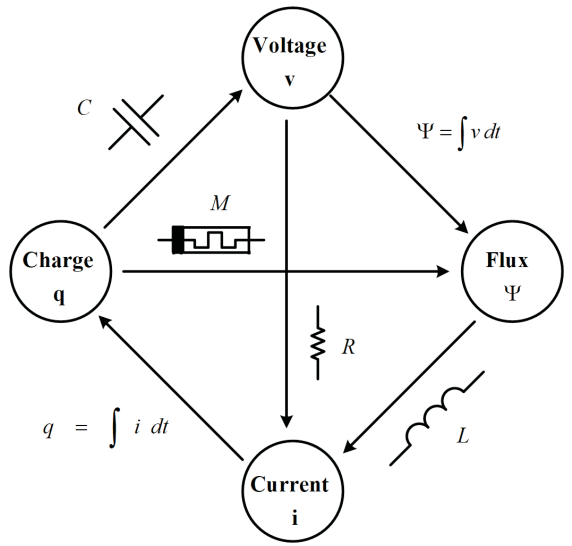

(a)
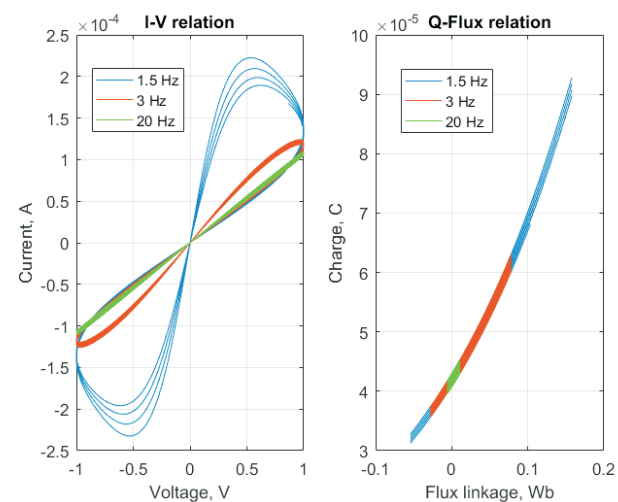

(b)

Fig. 1. (a) Relations between the basic electric quantities (current $i$, voltage $v$, electric charge $q$ and flux linkage $\Psi$ ), and the main one-port passive elements; (b) currentvoltage and charge-flux characteristics of idealized memristor for several different frequencies

the time integrals of the current (the charge) and of the voltage (the flux linkage) [2]. The system of equations describing the idealized memristor element is $[2,3]$ :

$$
\mid \begin{aligned}
& \frac{d x}{d t}=k i \\
& v=M(x) i
\end{aligned},
$$

where $x$ is a memristor state variable, proportional to the charge $q$ accumulated in the memristor, $k$ is a constant, $i$ is the current, $v$ is the voltage, and $M(x)$ has the measurement unit of resistance and is called incremental memristance $[2,3]$. It could be expressed as a derivative of the flux linkage with respect to the charge [3]:

$$
M(x)=\frac{d \varphi}{d q} .
$$

The current-voltage and the charge-flux relationships of an idealized memristor are presented in Fig. 1(b) for sinusoidal voltage with amplitude of $1 \mathrm{~V}$, frequencies of 1.5, 3 and $20 \mathrm{~Hz}$ and initial phase of $-60^{\circ}$. The current-voltage characteristic is a pinched hysteresis loop, which area decreases with increasing the frequency. The charge-flux relation is an increasing nonlinear curve, which tends to a straight line with increasing the frequency [3]. 


\subsection{Description of the titanium dioxide memristor}

The titanium dioxide memristor element has been created by Stanley Williams in Hewlett-Packard (HP) research labs [4]. A schematic of the memristor nanostructure is shown in Fig. 2(a). The electrodes of the element are made of titanium or other material. The structure of the memristor is founded on a thin layer of amorphous titanium dioxide. The first region of the memristor is created in the titanium dioxide nanostructure by doping with oxygen vacancies and the second one is made of pure titanium oxide [4]. The oxygen vacancies are positively charged. The doping process is called electroforming and is based on applying a constant voltage with a value of about $5 \mathrm{~V}$ [12]. Due to the derived electric field with high intensity, a partial evaporation of oxygen molecules near the anode occurs and oxygen vacancies appear in the left region of the memristor [4]. The stochiometric chemical formula of the doped region material is $\mathrm{TiO}_{2-z}$, where the index $z$ has a value between 0.02 and 0.05 [4]. The doped layer of the memristor nanostructure has low resistance, while the neighboring layer has very high resistance [4]. The length of the memristor nanostructure $D$ is between 3 and $10 \mathrm{~nm}$. The length of the doped layer could be altered by applying external electric field [4]. If a voltage with positive polarity is applied to the memristor element, then the positive potential on the anode repels the oxygen vacancies, and they start migration to the cathode of the element.

The length of the doped region increases and this process continues to the moment when the oxygen vacancies have reached the cathode. If a negative

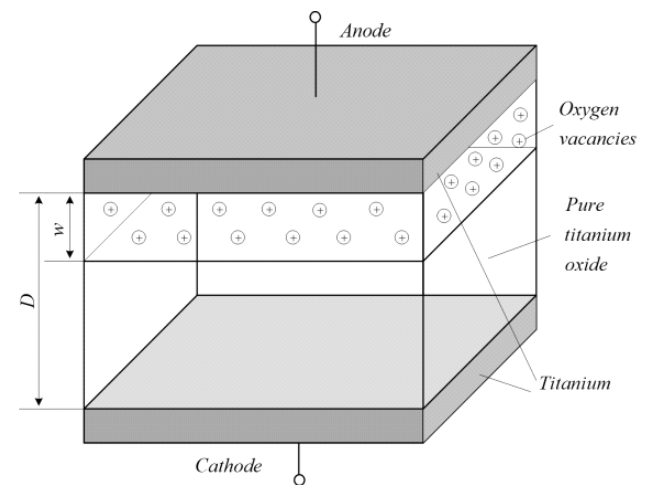

(a)

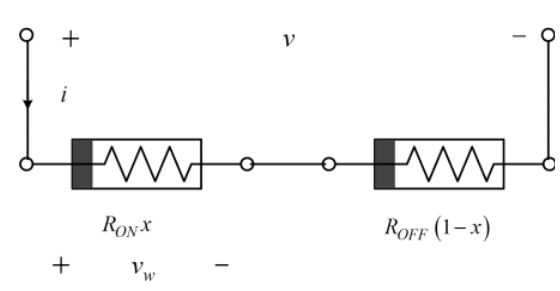

(b)

Fig. 2. (a) Memristor structure based on titanium oxide; (b) equivalent substituting circuit with state-dependent nonlinear resistors 
voltage is applied to the memristor, then the anode has a negative potential. It attracts the oxygen vacancies and the doped layer decreases [4, 22, 23]. The resistances of the doped and the un-doped regions of the memristor element depend on their instant lengths and on their specific resistances [4]. If the boundary between the doped and the un-doped layers of the memristor reaches the right border of the memristor nanostructure, then the length of the doped region is maximal. In this state, the memristor has a minimal resistance with a value of $100 \Omega$ [4]. This state of the memristor is known as a fully closed state and the respective resistance is known as ON-resistance $\left(R_{O N}\right)$. If the boundary between the doped and the un-doped layers of the memristor is on the left border of the memristor structure, then the whole region of the element is filled with pure titanium dioxide, and the resistance of the memristor element has a maximal value of $16 \mathrm{k} \Omega[4,22,24]$.

This state of the memristor is known as a fully open state, and its maximal resistance is known as the OFF-resistance $\left(R_{O F F}\right)$ [4]. The instant length of the doped region is denoted by $w$ and the length of the memristor is $D$ [4].

The memristor state variable $x$ is expressed as a ratio between the lengths of the doped layer $w$ and the size of the whole memristor element $D[4,25]$ :

$$
x=\frac{w}{D} .
$$

The doped and the un-doped parts of the memristor element are connected in a series, Fig. 2(a) [4]. A simplified equivalent circuit of the titanium dioxide memristor is shown in Fig. 2(b) for description of the memristor behavior in electric fields and for further derivation of the state-dependent current-voltage relation. The resistance of the respective memristor layer is proportional to its length and to the specific resistance. The resistance of the saturated region is proportional to the product of the ON-resistance and its respective length. The resistance of the un-doped region is proportional to its length and to the OFF-resistance of the memristor [4]. The equivalent resistance of the memristor element $R_{e q}$, according to the equivalent electric circuit, Fig. 2(b), is $[4,16,26]$ :

$$
R_{e q}=R_{O N} x+R_{O F F}(1-x) .
$$

The state variable $x$ determines the total resistance $R_{e q}$ of the memristor element. If a voltage signal $v$ is applied to the memristor element, the relationship between the memristor current $i$ and the applied voltage $v$ is expressed using the state-dependent Ohm's Law [4, 27]:

$$
v=i R_{e q}=i\left[R_{O N} x+R_{O F F}(1-x)\right] .
$$


The voltage across the doped layer of the memristor element $v_{w}$ is expressed using the Ohm's Law; it is a multiplication of the memristor current $i$, the resistance of the doped layer $R_{O N}$ and the state variable $x[4,12,28]$ :

$$
v_{w}=i R_{O N} x=i R_{O N} \frac{w}{D} .
$$

The intensity of the electric field in the doped layer of the memristor can be expressed as a ratio between the voltage across the saturated layer $v_{w}$ and its instantaneous length $w[4,12]$ :

$$
\vec{E}_{w}=\frac{v_{w}}{w}=\frac{i R_{O N} \frac{w}{D}}{w}=i \frac{R_{O N}}{D} .
$$

The relation between the velocity of the oxygen vacancies $v$, expressed as a time derivative of the instantaneous value of the length of the doped region $w$, and the electric field strength in the doped region of the memristor $E_{w}$ is [17]:

$$
\vec{v}=\frac{d w}{d t}=\frac{d}{d t}(x D)=D \frac{d x}{d t}=\mu \vec{E}_{w}=\mu i \frac{R_{O N}}{D},
$$

where $\mu=1 \times 10^{-14} \mathrm{~m}^{2} /$ (V.s) is the ionic mobility of oxygen vacancies in the titanium oxide material [4]. Equation (8) could be transformed as follows [4, $22,23]$ :

$$
\frac{d x}{d t}=\mu \frac{R_{O N}}{D^{2}} i=k i
$$

where $k$ is a constant, dependent only on the memristor parameters $\mu, R_{O N}$ and $D$ [4]. Equation (9) is the fundamental state differential equation of the titanium dioxide memristor element with linear ionic dopant drift $[4,23]$. Equations (2) and (9) completely describe the behavior of the memristor element in electric fields [4] and they are combined to form system of equations (10):

$$
\mid \begin{aligned}
& v=i\left[R_{O N} x+R_{O F F}(1-x)\right] \\
& \frac{d x}{d t}=k i
\end{aligned} .
$$

After expression of the current from the first equation of system (10) as a function of the voltage and of the state variable, substituting in the second equation of (10), separating the variables $x$ and $v$, and integration the following expression is derived:

$$
\int_{x_{0}}^{x}\left[\left(R_{O N}-R_{O F F}\right) y+R_{O F F}\right] d y=k \int_{0}^{t} v d \tau=k \Psi .
$$


After integrating and solving the obtained expression with respect to the state variable, the following solutions (12) are derived [17]:

$x_{1,2}=\frac{-R_{O F F} \pm \sqrt{R_{O F F}^{2}-4 \frac{\left(R_{O N}-R_{O F F}\right)}{2}\left[k \Psi+\frac{\left(R_{O N}-R_{O F F}\right)}{2} x_{0}^{2}+R_{O F F} x_{0}\right]}}{R_{O N}-R_{O F F}}$.

According to physical considerations only the second solution satisfies the condition $0<x<1$. It is obvious that the state variable $x$ is proportional to the time integral of the applied voltage. This fact confirms the memory effect of the considered memristor. Using the fact that $R_{O N} \ll R_{O F F}$, the following approximation could be applied [17]:

$$
R_{O N}-R_{O F F} \approx-R_{O F F} .
$$

By applying this approximate expression in solutions (12), the state variable $x$ could be expressed by a simplified formula [17]:

$$
x=1-\sqrt{1+\frac{2 k}{R_{O F F}} \int_{0}^{t} v d \tau-x_{0}^{2}+2 x_{0}}
$$

If the time integral of the memristor voltage is replaced by the equivalent flux linkage $\Psi$, the following expression could be derived [17]:

$$
x=1-\sqrt{1+\frac{2 k}{R_{O F F}} \Psi-x_{0}^{2}+2 x_{0}} .
$$

Formula (15) describes the relationship between the state variable and the flux linkage and it is also known as state-flux relationship [17]. After substitution of expression (15) into ratio (3) the current-voltage relationship of the memristor element is derived:

$$
i=\frac{v}{\left[\left(R_{O N}-R_{O F F}\right)\left[1-\sqrt{1+\frac{2 k}{R_{O F F}} \int_{0}^{t} v d \tau-x_{0}^{2}+2 x_{0}}\right]+R_{O F F}\right]} .
$$

Observing relationship (16) it could be concluded that the memristor current depends not only on the applied voltage but on the time integral of the voltage as well. This confirms the memory effect and the pinched hysteresis loop of the $i-v$ characteristic. 


\subsection{Memory effect of the memristor element in electric fields}

This effect observed at the memristor, operating in electric fields is visually expressed in the next Fig. 3(a, b). In the first case the applied memristor voltage is a sequence of rectangular pulses with positive polarity $[29,30]$. The duty cycle of the applied pulse sequence is $50 \%$. If a positive voltage impulse is applied to the memristor, then its state variable $x$ increases, and the memristance $M$ (known also as the resistance of the memristor), proportional to the state variable $x$, decreases. In the pauses between the pulses the memristor voltage is zero and the state variable of the memristor $x$ retains its previous value. In the time diagram, the state variable is presented with horizontal segments.

The observed effect confirms the memorizing property of the memristor [4]. When a new positive pulse is applied, the state variable $x$ increases again, starting from its previously reached value. In Figure 3(b) the applied voltage is presented as a sequence of positive and negative impulses. If the respective pulse is negative then the state variable decreases. It could be concluded that the memristor state and resistance could be changed by external voltage pulses with different polarity. The change of the state variable and the respective memristance is proportional to the time integral and henceforth to the respective duration of the applied voltage pulses. The described properties and characteristics of the memristor element are valid for the idealized case, when the state variable $x$ does not reach its limiting values. In this case the memristor operates in a soft-switching mode $[4,17]$.

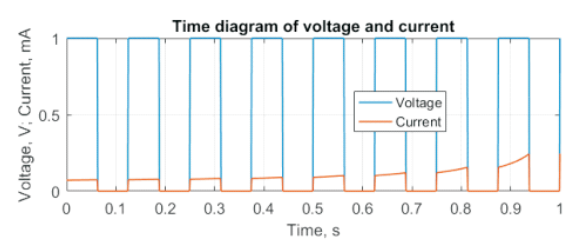

Time diagrams of the flux and state

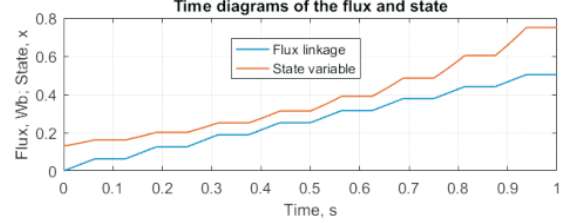

(a)

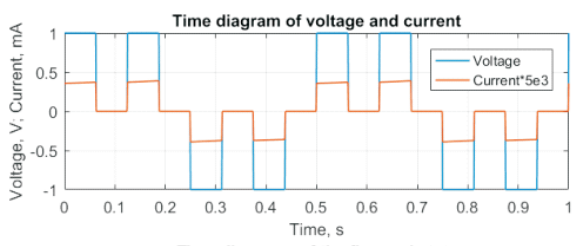

Time diagrams of the flux and state

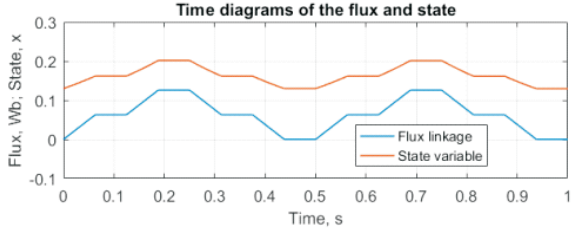

(b)

Fig. 3. (a) Time diagrams of the memristor voltage, current, state and flux linkage for a sequence of positive impulses; (b) the respective diagrams for pulses with different polarities 


\subsection{Present and future potential applications of the memristors}

Due to their very useful properties - memory effect [4], long retention intervals [17], low power consumption [22, 23], the memristors have many applications - in reconfigurable analogue and digital circuits $[17,26]$, neural networks $[7,19,26,27,28]$, computer memories $[2,14,26]$ and other promising potential capabilities for applications in electronics [6, 26, 31].

If the memristor operates with high-frequency signals then the state variable has almost a constant value and the memristance is also a constant $[3,32$, 33]. In such cases the memristor could be applied in linear electronic devices, as filters [22], amplifiers [34], integrators [35, 36] and many other circuits for linear operations. If an external impulse with a long duration and a high level is applied to the memristor then its state and the respective resistance are changing till stopping the external signal. Then the considered analogue or digital device continues its operation without changing the memristance. In this way the memristor could be used in linear electronic schemes if the change of several parameters is needed.

The memristor could be applied in nonvolatile memories [6, 23]. For storing a bit of information a positive pulse with high level and long duration is used. Then the memristor state and resistance are changed and the new value of the memristance is proportional to the written logical information. For reading the information stored in the memristor there are two possible variants - applying a sequence of two equivalent pulses with different polarities, or using a pulse with nominal duration and a level lower than the memristor activation threshold. In both the considered cases the memristor state and the corresponding stored information do not change during the reading processes [17]. For erasing the information stored in the memristor a negative pulse with nominal level and a long duration is applied. Then the memristor state obtains its initial value derived before the writing procedure [4, 17, 23].

The change of the memristor state and respective resistance and the possibility for retaining its value after turning the source off could be used for application of the memristors in the synapses of the artificial neural networks $[25,26,27]$. Several different schematics of memristor-based synapses exist in the scientific literature - anti-series memristor circuits, bridge memristor circuits [26], and single memristor circuits [25], bridge resistor-memristor circuits with one and two anti-parallel memristors [37]. The change of the synaptic weights of the synapses is made in the learning phase of the neural network [26]. This change is made by applying voltage pulses with long duration and a level higher than the memristor activation threshold. In the phase of applying of the input signal to the neural network this signal has to be with levels lower 
than the activation threshold [17]. Then the resistances of the memristors in the synapses are with constant values till applying the next tuning pulse for adjusting the synaptic weights.

The memristors could be applied in oscillator circuits [32, 33]. If the memristors are used for tuning the amplitude or the frequency of the output signal of the generator then they must operate in a state near to soft switching mode with low-voltage signals and their state must not be changed. The change of the frequency or the output voltage level could be made by applying external voltage pulses for changing the resistance of the memristors. This mode is used in sinusoidal generators [32]. If the memristor operates with high-level voltage signals then the output voltage signal is non-sinusoidal one and this mode could be used for relaxation pulse oscillators [33].

In the last several years the memristorc are used for constructing of logic gates [38, 39]. In such devices the memristors operate in hard-switching [17].

According to many investigations it is established that the axons in the biological neural systems have memristive behavior [40]. One of the future possible applications of the memristors could be their inclusion in the place of the axons of the artificial neurons in the multilayer neural networks [26, 40].

Many investigations associated with the in-memory computing are published in the scientific literature in the last several years [31]. These effective calculations in the computer memories operating also as processing modules could be prepared with the use of memristor elements [31].

\section{A REVIEW AND COMPARISON OF THE BASIC STANDARD AND MODIFIED MEMRISTOR MODELS}

In this Section the basic classical and modified by the author memristor models are considered, paying attention to their main properties and advantages. All of them could be represented by the following system of equations $[4,11]$ :

$$
\mid \begin{aligned}
& v=i\left[R_{O N} x+R_{O F F}(1-x)\right] \\
& \frac{d x}{d t}=k i f(x, i)
\end{aligned},
$$

where $f(x)$ is the applied window function, used for limitation of the state variable in the interval $[0,1]$ and for representation the boundary effects [17].

\subsection{Strukov-Williams memristor model}

This memristor model uses the system of equations (17) in a combination with the Strukov-Williams window function (18) [4]: 


$$
f_{S W}(x)=1-(2 x-1)^{2}=4 x(1-x) .
$$

The presented window function $f_{s w}(x)$ is appropriate for limitation of the state variable $x$ but unfortunately it could not completely represent the boundary effects [17]. It is a special case of the next Joglekar window function [15].

\subsection{Joglekar memristor model}

This memristor model is described by the system of equations (17) in a combination with the Joglekar window function $f_{J}(x)(19)$ [15]:

$$
f_{J}(x)=1-(2 x-1)^{2 p},
$$

where $f_{J}(x)$ is the window proposed by Joglekar $[15,17]$. This model is also unable to represent the boundary effects for hard-switching mode [17].

\subsection{Biolek memristor model}

The Biolek model is described by the system of equations (17) in a combination with (20) [16]:

$$
f_{B}(x, i)=1-[x-\operatorname{stp}(-i)]^{2 p},
$$

where $f_{B}(x, i)$ is the Biolek window function [16]. Due to the use of this special window function the Biolek model is able to represent the boundary effects [17]. Sometimes the use of the non-differentiable step function could lead to convergence problems in PSpice environment [9].

\subsection{Boundary condition memristor model}

This memristor model is given by the system of equations (17) in a combination with (21) [17]:

$$
f_{B C M}(x, v)= \begin{cases}1, & x \in(0,1) \\ 1, & x=0 \quad \& v \geq v_{t h r} \\ 0, & x=0 \& v<v_{t h r} \\ 1, & x=1 \& v<-v_{t h r} \\ 0, & x=1 \& v \geq-v_{t h r}\end{cases}
$$

where $f_{B C M}(x, v)$ is the boundary condition memristor window. This window function successfully represents the boundary conditions [17]. The model represents a linear dopant drift. 


\subsection{Lehtonen-Laiho memristor model}

Unlike the previous memristor models described above this model is a highly nonlinear one [19]. It uses the standard Biolek window and could represent the boundary effects for hard-switching mode [17, 19]. It is appropriate for application in high-frequency devices [19] operating with high-level voltage signals. Its describing system of equations is [19]:

$$
\mid \begin{aligned}
& i=x^{n} \beta \sinh (\alpha v)+\chi[\exp (\gamma v)-1] \\
& \frac{d x}{d t}=a f_{B}(x) v^{m}
\end{aligned},
$$

where $n, m, a, \alpha, \beta, \gamma$ and $\chi$ are parameters for fitting the model [19].

\subsection{Modified window functions}

The basic modifications proposed by the author $[10,36,41,42]$ are motivated by the established dependence between the nonlinearity of the ionic dopant drift and the applied memristor voltage [10, 26]. For expression of this dependence in the standard window functions the integer exponent $p$ is replaced by a function of the applied voltage $p(v)[10,41]$ :

$$
p(v)=\operatorname{round} \frac{a}{b+|v|},
$$

where $a$ and $b$ are fitting parameters and the function "round" is used for deriving integer result $[24,26]$. The function (23) is applied in the modified Joglekar and "Biolek 1" memristor models and their improved versions are derived $[10,41]$. A comparison between them is presented in Table 1.

For more realistic representation of the nonlinear dopant drift an additional sinusoidal component [42] is used in the modified "Biolek 2" memristor model window function [16]:

$$
f_{B M}(x, i)=\frac{1-[x-s t p(-i)]^{2 p}+m \sin ^{2}(\pi x)}{1+m} .
$$

The next basic modification proposed for increasing the model nonlinearity is combining the modified Biolek and Joglekar windows [10, 36]:

$$
f_{B J M}(x, v)=\frac{f_{B}(x, v)+f_{J}(x, v)}{2} .
$$

The derived mixed window function is applied in a memristor model described in $[10,36]$ with a combination of activation thresholds. 


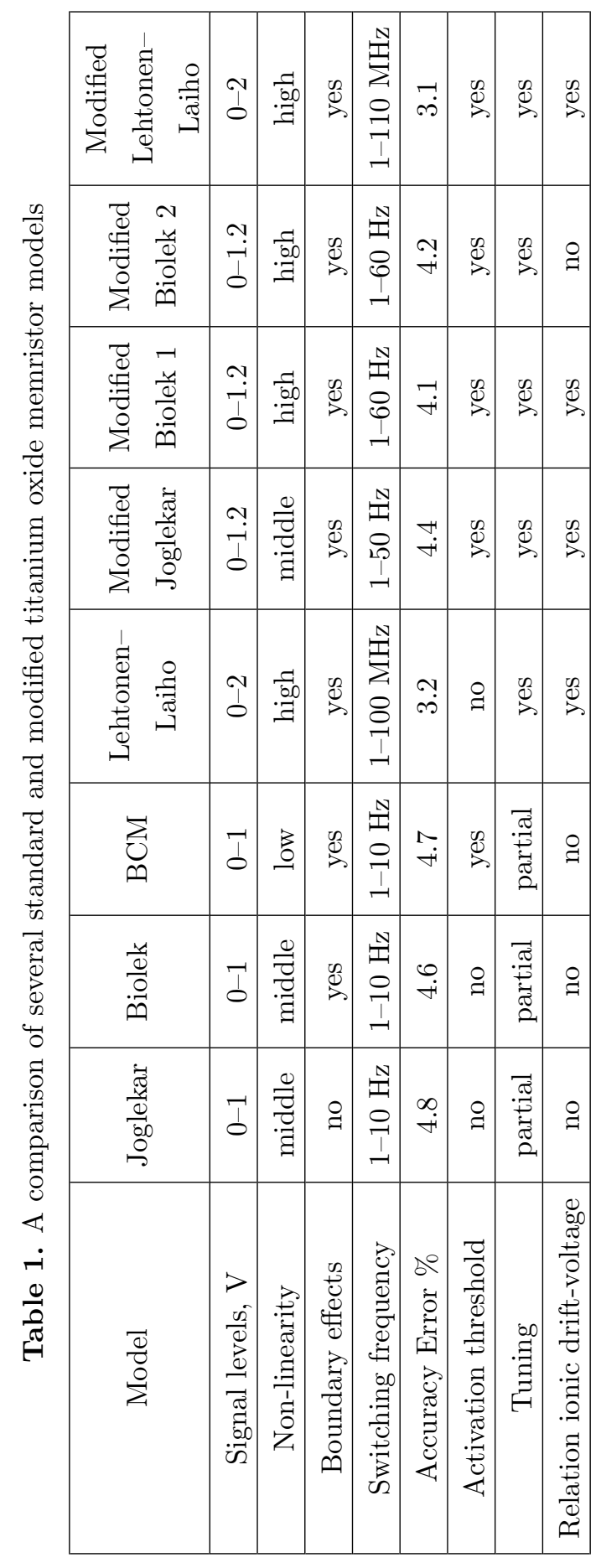




\subsection{Avoiding convergence problems in PSpice environment by replacing the step function with its tangent hyperbolic differentiable analogue}

For derivation of stable PSpice memristor library models and for avoiding convergence problems the standard step function in the Biolek classical window could be replaced by its continuous and differentiable analogue. In [9] this is made by the use of exponential function. In [21] the author proposed applying of a differentiable analogue of the step function based on tangent hyperbolic function in the modified Biolek window function with a fitting parameter $r$ $[21,42]$ :

$$
g(i)=\frac{1+\tanh (r i)}{2} .
$$

The proposed by the author modifications to the standard models lead to more realistic representation of the memristor behavior in electric circuits which allows using the models for high-level and high-frequency signals $[10$, $36,41]$.

\subsection{A comparison of the considered memristor models}

In Table 1 a comparison of the considered standard and modified memristor models is made using several important criteria - operating signal levels, nonlinearity, and expression of the boundary effects for hard-switching mode, switching frequency, accuracy, activation thresholds, and possibility of tuning, expression the dependence of the nonlinear ionic drift on the applied voltage. All the considered standard models with exception of the Lehtonen-Laiho model are appropriate for low-frequency and low-level signals. The modified by the author models are with improved characteristics and they could be used for electronic circuits investigation for high-level and high-frequency signals. The modified models are tunable and could be adjusted according to experimentally derived current-voltage characteristics of transition metal oxide-based memristors [10].

\section{CONCLUSION}

The memristors are very important and promising electronic elements with many present and potential future applications in reconfigurable devices, oscillator circuits, computer memories, neural networks, in-memory computing devices and many others. The basic types of memristors, their structure and 
principle of operation are discussed in the paper. Special attention is paid to their modeling and the associated problems due to their high complexity. In order to compensate several problems in the existing standard memristor models the author has proposed several improvements in the window function, applying dependence between the dopant drift nonlinearity and the voltage and adding additional sine component in the windows. For avoiding convergence problems the author has proposed replacement of the step function by its analogue based on the tangent hyperbolic function. In this way the modified models could be successfully used for analysis of complex electronic schemes for high-level and high-frequency signals.

\section{REFERENCES}

[1] G. Dearnaley, A. Stoneham and D. Morgan, Electrical phenomena in amorphous oxide films, Rep. Prog. Phys. (1970) 33 1129-1191.

[2] L. ChuA, Resistance switching memories are memristors, Applied physics A, Material science and processing, January (2011) 765-783, DOI https://doi.org/10.1007/s00339-011-6264-9.

[3] L. Chua, Memristor - The Missing Circuit Element, IEEE Transactions on Circuit Theory (1971) 18 507-519, DOI: 10.1109/TCT.1971.1083337.

[4] D. Strukov, G. Snider, D. Stewart and R. S. Williams, The Missing Memristor Found, Nature Letters (2008) 453 80-83, https://doi.org/10.1038/nature06932.

[5] Z. Biolek, D. Biolek and V. Biolková, Computation of the Area of Memristor Pinched Hysteresis Loop, IEEE Transactions on Circuits and Systems II: Express Briefs (2012) 59 (9), DOI: 10.1109/TCSII.2012.2208670.

[6] J. Yang, M. Zhang, M. Pickett, F. Miao, J. Strachan, W. Li, W. Yi, D. Ohlberg, B. Choi, W. Wu, J. Nickel, G. Ribeiro and R. Williams, Engineering nonlinearity into memristors for passive crossbar applications, Appl. Phys. Lett. (2012) 100 (113501) 1-5, https://doi.org/10.1063/1.3693392.

[7] Y. Chen, G. Liu, C. Wang, W. Zhang, R. Li and L. Wanga, Polymer memristor for information storage and neuromorphic applications, Mat. Horiz. (2014) 489-506, DOI: 10.1039/C4MH00067F.

[8] H. Abdalla And M. Pickett, SPICE modeling of memristors, IEEE International Symposium on Circuits and Systems (2011) 1832-1835, DOI: 10.1109/ISCAS.2011.5937942.

[9] A. Ascoli, R. Tetzlaff and L. Chua, Robust Simulation of a TaO Memristor Model, Radio engineering (2015) 24 (2) 384-392, DOI: 10.13164/re.2015.0384. 
[10] V. Mladenov, Advanced Memristor Modeling, Dissertation for awarding the scientific degree "Doctor of Sciences", Technical University of Sofia (2019).

[11] E. Linn, A, Siemon, R. Waser and S. Menzel, Applicability of WellEstablished Memristive Models for Simulations of Resistive Switching Devices, IEEE Trans. Circ. Syst. (2014) 2402-2410, DOI: 10.1109/TCSI.2014.2332261.

[12] J. Borghetti, D. Strukov, M. Pickett, J. Yang, D. Stewart and R. S. Williams, Electrical transport and thermometry of electroformed titanium dioxide memristive switches, Journal of Applied Physics (2009) 106 (124504) 1-5, https://doi.org/10.1063/1.3264621.

[13] Z. Hu, Q. Li, M. Li, Q. Wang, Y. Zhu, X. Liu, X. Zhao, Y. LiU AND S. Dong, Ferroelectric memristor based on $\mathrm{Pt} / \mathrm{BiFeO} 3 / \mathrm{Nb}$ doped SrTiO3 heterostructure, Appl. Phys. Lett. (2013) 102 1-5, https://doi.org/10.1063/1.4795145.

[14] H. Mostafa And Y. Ismail, Process Variation Aware Design of Multi-Valued Spintronic Memristor-Based Memory Arrays, IEEE Trans. Semicond. Manuf. (2016) 145, DOI: 10.1109/TSM.2016.2551224.

[15] Y. Joglekar and S. Wolf, The Elusive Memristor: Properties of Basic Electrical Circuits, European Journal of Physics (2009) 30 661-675.

[16] Z. Biolek, D. Biolek And V. Biolkova, SPICE Modeling of Memristor with Nonlinear Dopant Drift, Radio engineering (2009) 18 210-214.

[17] F. Corinto And A. Ascoli, A Boundary Condition-Based Approach to the Modeling of Memristor Nanostructures, IEEE Transactions on Circuits and Systems (2012) 59 2713-2726.

[18] A. Ascoli, R. Tetzlaff, Z. Biolek, Z. Kolka, V. Biolkovà and D. BiOLEK, The Art of Finding Accurate Memristor Model Solutions, IEEE J. Emerg. Sel. Top. Circuits Syst. (2015) 5 133-142, DOI:10.1109/JETCAS.2015.2426493.

[19] E. Lehtonen And M. LAiHo, CNN using memristors for neighborhood connections, in: IEEE Proceedings of $12^{\text {th }}$ (CNNA), Berkeley, CA, USA, 3-5 February 2010, pp. 1-4.

[20] V. Mladenov, A New Simplified Model for $\mathrm{HfO}_{2}$-Based Memristor, in: IEEE Proceedings of $8^{\text {th }}$ MOCAST, 2019, 1-4, DOI: 10.1109/MOCAST.2019.8741953.

[21] V. Mladenov, A Modified Tantalum Oxide Memristor Model for Neural Networks with Memristor-Based Synapses (submitted for presentation and publishing in MOCAST 2020, Germany, http://www.ids.unibremen.de/conf/mocast2020/index.html.

[22] A. Ascoli, R. Tetzlaff, F. Corinto, M. Mirchev and M. Gilli, Memristor-based filtering applications, IEEE $201314^{\text {th }}$ LATW, DOI: 10.1109/LATW.2013.6562672.

Engineering Sciences, LVII, 2020, No. 1 
[23] Z. Jiang, Y. Wu, S. Yu, L. Yang, K. Song, Z. Karim and H.-P. Wong, A Compact Model for Metal-Oxide Resistive Random-Access Memory with Experiment Verification, IEEE Trans. Electron. Devices (2016) 63 1884-1892.

[24] P. Mazumder, S. Kang and R. Waser, Memristors: devices, models, and applications, Proceedings of the IEEE (2012) 100 (6), DOI: 10.1109/JPROC.2012.2190812.

[25] A. Walsh, R. Carley, O. Feely and A. Ascoli, Memristor circuit investigation through a new tutorial toolbox, IEEE ECCTD (2013) 1-4, DOI: 10.1109/ECCTD.2013.666226.

[26] M. Sah, C. Yang, H. Kim, T. Roska and L. Chua, Memristor Bridge Circuit for Neural Synaptic Weighting, in: Proc. $13^{\text {th }}$ CNNA, IEEE, Italy, 2012, pp. 1-5, DOI:10.1109/CNNA.2012.6331434.

[27] I. Ebong and P. Mazumder, CMOS and Memristor-Based Neural Network Design for Position Detection, Proc. IEEE (2012) 100 (6) 2050-2060, DOI: 10.1109/JPROC.2011.2173089.

[28] M. Prezioso, F. Merrikh-Bayat, B. Hoskins, G. Adam, K. Likharev AND D. STRukov, Training and operation of an integrated neuromorphic network based on metal-oxide memristors, Nature, May (2015) 521 61-64, https://doi.org/10.1038/nature14441.

[29] W. PALM, Introduction to MATLAB for engineers, McGraw-Hill (2011), ISBN 978-1-259-01205-1.

[30] M. RASHID, Introduction to PSpice using OrCAD for circuits and electronics, Prentice Hall, $3^{\text {rd }}$ Edition (2004), ISBN 0-13-101988-0.

[31] M. Lebdeh, H. Abunahla, B. Mohammad and M. Qutayri, An Efficient Heterogeneous Memristive XNOR for In-Memory Computing, IEEE Trans. Circ. Syst. (2017) 1-11, DOI 10.1109/TCSI.2017.2706299.

[32] B. BAO, Z. LiU AND J. XU, Steady periodic memristor oscillator with transient chaotic behaviors, IEEE Electronics Letters (2010) 46 (3) 237-238, DOI: 10.1049/el.2010.3114.

[33] M. Iтон AND L. ChUA, Memristor Oscillators, International Journal of Bifurcation and Chaos (2008) 18 (11) 3183-3206, https://doi.org/10.1142/S0218127408022354.

[34] T. Wey And W. Jemison, Variable gain amplifier circuit using titanium dioxide memristors, IET Circuits, Devices 83 Systems (2011) 5 (1) 59-65, DOI: 10.1049/iet-cds.2010.0210.

[35] S. Kirilov and V. Mladenov, Integrator device with a memristor element, in: $7^{\text {th }}$ International Conference (MOCAST), 2018, DOI: 10.1109/MOCAST.2018.8376656. 
[36] V. Mladenov and S. Kirilov, A Memristor Model with a Modified Window Function and Activation Thresholds, IEEE (ISCAS), 2018, DOI: 10.1109/ISCAS.2018.8351429.

[37] V. Mladenov, S. Kirilov, Learning of an Artificial Neuron with ResistorMemristor Synapses, ANNA'18, IEEE, VDE (2018), Print ISBN: 978-3-80074756-6.

[38] E. Gale, B. De Lacy Costello and A. Adamatzky, Boolean Logic Gates from a Single Memristor via Low-Level Sequential Logic (Eds G. Mauri, A. Dennunzio, L. Manzoni, A. E. Porreca) Unconventional Computation and Natural Computation, Lecture Notes in Computer Science, Springer, Berlin, Heidelberg (2013) 7956, https://doi.org/10.1007/978-3-642-39074-6_9.

[39] Q. Xia, W. Robinett, M. Cumbie, N. Banerjee, T. Cardinali, J. Yang, W. Wu, X. Li, W. Tong, D. Strukov, G. Snider, G. Medeiros and R. S. Williams, Memristor-CMOS Hybrid Integrated Circuits for Reconfigurable Logic, Nano Lett. (2009) 9 (10) 3640-3645, https://doi.org/10.1021/nl901874j.

[40] M. Sah, H. Kim and L. Chua, Brains Are Made of Memristors, IEEE Circuits and Systems Magazine (2014) 14 (1) 12-36, DOI: 10.1109/MCAS.2013.2296414.

[41] V. Mladenov and S. Kirilov, A Nonlinear Memristor Model with Activation Thresholds and Variable Window Functions, in: $15^{\text {th }}$ International Workshop on Cellular Nanoscale Networks and their Applications, CNNA 2016, IEEE, VDE, Print ISBN: 978-3-8007-4252-3.

[42] V. Mladenov and S. Kirilov, A Nonlinear Drift Memristor Model with a Modified Biolek Window Function and Activation Threshold, Electronics (2017) 6 (77) 1-15, DOI:10.3390/electronics6040077. 